\title{
Satisfacción de usuarias externas en la atención gineco-obstétrica, Instituto Hondureño de Seguridad Social, Tegucigalpa, 2016-2017.
}

\author{
Satisfaction of the external users in gyneco-obstetric care, Instituto Hondureño de Seguridad Social, \\ Tegucigalpa, 2016-2017.
}

Ana Ligia Chinchilla, ${ }^{1}$ Jessica Sabonge. ${ }^{2}$

\begin{abstract}
1Doctora en Medicina, Especialista en Ginecología y Obstetricia; Servicio de Ginecología y Obstetricia, Clínica Periférica No. 1, Instituto Hondureño de Seguridad Social; Departamento de Ciencias Morfológicas, Facultad de Ciencias Médicas UNAH.

2Doctora en Medicina, Médico Residente periodo 2016-2018, Posgrado de Ginecología y Obstetricia, Facultad de Ciencias Médicas UNAH; actualmente Especialista en Ginecología y Obstetricia, PorSalud, Centro Hondureño de Ginecología y Obstetricia; Tegucigalpa.
\end{abstract}

\begin{abstract}
RESUMEN. Antecedentes: El concepto de calidad de atención implica que los pacientes estén informados y compartan la toma de decisiones para su atención. Objetivo: Establecer el nivel de satisfacción en la atención brindada en el Servicio de Ginecología y Obstetricia, Clínica Periférica No.1, Instituto Hondureño de Seguridad Social (IHSS), Tegucigalpa, 2016-2017. Métodos: Se utilizó la Encuesta de Satisfacción de Usuarios del Régimen del Seguro de Atención de Salud Modificada, creada por el IHSS (Año 2014). Fue aplicada, previo consentimiento informado escrito, por médicos residentes del Posgrado de Ginecología y Obstetricia, Facultad de Ciencias Médicas UNAH. Resultados: Se encuestó un total de 350 pacientes. El grupo de edad más frecuente fue 18-35 años $61.0 \%$ (215). Los trámites de admisión fueron percibidos como rápidos por 46.0\% (163). El 92.0\% (322) y 84.0\% (295) de las pacientes expresaron encontrarse satisfechas por la atención brindada por el personal médico y de enfermería, respectivamente. La información brindada acerca de los cuidados después de la consulta médica fue percibida como buena $82.9 \%$, (290), regular $12.3 \%$ (43) y mala $4.9 \%$ (17). La privacidad brindada en la consulta médica fue percibida como buena $70.3 \%$ (246), regular 21.1\% (95) y mala $2.6 \%$ (9). En cuanto a la comodidad ofrecida durante la consulta médica, fue percibida como buena $82.9 \%$, (290), regular $13.7 \%$ (48) y mala $3.4 \%$ (12). Discusión: Una proporción importante de las usuarias externas entrevistadas consideró la atención brindada como buena y refirió que le gustaría que se ampliara la oferta de servicios. Es necesario promover la mejora continua de la atención en salud.

Palabras Clave: Accesibilidad a los servicios de salud, Atención ambulatoria, Calidad de atención de salud, Estudios de evaluación, Prestación de atención de salud.
\end{abstract}

\section{INTRODUCCIÓN}

Desde hace más de dos décadas, el concepto de calidad de atención ha incluido un enfoque tanto individual como poblacional, asegurando el otorgamiento de servicios de salud con base en la mejor evidencia científica disponible y con énfasis en los resultados deseables en salud. Este concepto implica que los pacientes estén informados y compartan la toma de decisiones para su atención. ${ }^{1}$ Asegurar el derecho a la salud de calidad es requisito para acortar las brechas poblacionales y reducir así la pobreza y la desigualdad. ${ }^{2}$ Hasta mediados del siglo pasado, la calidad era un tema eminentemente industrial y asociado a la competencia de bienes de consumo, lo que obligó a los empresarios a ofrecer mejores productos para ganar el mercado y mantenerse operando. Fue hasta el surgimiento de la competencia entre las empresas de servicios, que inicia la cultura de la calidad al cliente en este sector. ${ }^{3}$

El impulso de la teoría de la calidad en los servicios de salud empezó poco después de su inicio en los Estados Unidos

Recibido: 30-1-2019; Aceptado para publicación 30-6-2019

Dirección para correspondencia: Dra. Jessica Sabonge

Correo electrónico: jessicanad10@hotmail.com

Conflictos de interés. Los autores declaran no tener conflictos de interés en relación a este artículo.

DOI: https://doi.org/10.5377/rmh.v87i2.11901 y algunos países de Europa. El concepto de calidad en la atención médica de acuerdo a la definición de Avedis Donabedian (padre de la calidad) es: "el tipo de atención que maximiza el bienestar del paciente, después de tener en cuenta el balance de pérdidas y ganancias esperadas, contemplando el proceso de atención en todas sus partes". ${ }^{3}$ La propia Organización Mundial de la Salud (OMS), la ha definido como "una atención sanitaria de alta calidad es la que identifica las necesidades de salud de los individuos o de la población de una forma total y precisa y destina los recursos necesarios (humanos y otros) a estas necesidades, de forma oportuna y tan efectiva como el estado actual del conocimiento lo permite" 3,4 También la atención de calidad en los servicios médicos orientados para la mujer son importantes, así lo demuestran la pertinencia de diagnosticar la situación en el desempeño y la infraestructura de los servicios de salud de primer nivel que atienden durante la maternidad a la mujer. Al mismo tiempo, así como en el caso de México, se constata la necesidad de poder contar con un "Índice de Calidad de la Atención Materna" para el primer nivel, para tener un parámetro pertinente de comparación. ${ }^{5,6}$

La Seguridad Social en nuestro país cuenta con servicios de atención ambulatoria y hospitalaria. Si bien no existe un paquete definido de servicios mínimos, los programas de atención primaria incluyen: atención integral de la familia (niños 
adolescentes, mujeres, hombres y adultos mayores), control de enfermedades de transmisión vectorial (dengue, Chagas, leishmaniasis, malaria, leptospirosis) y de transmisión sexual, y otros programas dirigidos a atender las necesidades en materia de enfermedades crónico-degenerativas, salud mental y promoción de la salud, entre otras. ${ }^{7}$ Este estudio se realizó con el objetivo de establecer el nivel de satisfacción en la atención brindada en el Servicio de Ginecología de la Clínica Periférica No. 1 del Instituto Hondureño de Seguridad Social (IHSS) en Tegucigalpa.

\section{METODOLOGÍA}

Se realizó un estudio descriptivo transversal sobre una muestra por conveniencia de las pacientes que asistieron a la Consulta Externa de Ginecología y Obstetricia en la Clínica Periférica No. 1 del IHSS, Tegucigalpa. El estudio se realizó en el periodo de diciembre 2016 y enero 2017.

Se utilizó la Encuesta de Satisfacción de Usuarios del Régimen del Seguro de Atención de Salud, creada por el IHSS (Año 2014), modificando algunas de sus preguntas para adaptarla a la atención brindada de forma ambulatoria. La encuesta incluyó preguntas del proceso de atención desde la inscripción, atención de enfermería, atención médica, valoración del espacio físico de consulta, otros servicios como farmacia, planificación familiar, ultrasonido, limpieza, etc. Se utilizó una medida de gradación de buena, regular y mala. La encuesta fue aplicada por los médicos residentes del Posgrado de Ginecología y Obstetricia, Facultad de Ciencias Médicas UNAH, que estaban realizando una pasantía por la clínica, previo entrenamiento en su aplicación. Al momento de la entrevista, no se identificaron como médicos ante las usuarias.

Se creó una base de datos en Epilnfo versión 7.2.3.1 (CDC, Atlanta, EUA). Los resultados se presentan como frecuencias y porcentajes de las variables estudiadas. El estudio fue aprobado por el Comité de Etica en Investigación de la Gerencia de Docencia e Investigación, IHSS. Las pacientes fueron invitadas a participar en el estudio mediante consentimiento informado escrito.

\section{RESULTADOS}

Durante el periodo de estudio, el Servicio de Ginecología y Obstetricia de la Clínica Periférica No. 1 brindó atención a 3,800 pacientes. De este total, $350(9.2 \%)$ aceptaron participar de un grupo de pacientes abordadas tanto en horario matutino como vespertino. Las razones por las cuales algunas de las pacientes no aceptaron participar, incluyeron falta de tiempo, permiso limitado en el trabajo y sin interés en el tema. De este total de 350 participantes, 237 (68.0\%) fueron atendidas en el horario matutino $(7 \mathrm{am}$ a $1 \mathrm{pm}), 12(3.0 \%)$ eran pacientes menores de 18 años de edad, $215(61.0 \%)$ tenían entre 18 y 35 años y $123(35.0 \%)$ eran mayores de 35 años;
$63(18.0 \%)$ pacientes habían cursado la escuela primaria, 151 $(43.0 \%)$ secundaria y $136(39.0 \%)$ eran universitarias. Las pacientes que se encontraban embarazadas al momento del estudio fueron 138 (39.4\%).

En relación a las percepciones expresadas por las pacientes acerca de los servicios de admisión brindados, 156 (44.6\%) consideraron que los servicios prestados en general fueron rápidos, $139(39.7 \%)$ regulares y $55(15.7 \%)$ lentos. El tiempo de espera para recibir la atención médica fue percibido como rápido para $163(46.6 \%)$, regular $145(41.4 \%)$ y lento $42(12.0 \%)$. En el Cuadro 1 se presentan las percepciones acerca de otros servicios brindados. La información acerca de los cuidados después de la consulta médica, la privacidad y comodidad durante la consulta médica fueron percibidas como buenas por $290(82.9 \%), 246(70.3 \%)$ y $290(82.9 \%)$, respectivamente. La limpieza en el consultorio y el aseo de los sanitarios fueron percibidos como buenos por $285(81.4 \%)$ y $201(57.4 \%)$, respectivamente. El servicio ofrecido por el Departamento de Farmacia fue considerado como malo por 159 (45.4\%). La orientación por parte del personal de enfermería y la explicación acerca del estado de salud de parte del médico fueron percibidas como buenas por $244(69.7 \%)$ y $280(80 \%)$, respectivamente.

De las 350 pacientes, $224(69.7 \%)$ recibieron consejería familiar y la calificaron como buena $107(47.8 \%)$, regular $20(8.9 \%)$ y mala $8(3.6 \%)$. De las 350 pacientes, $140(40.0 \%)$ pacientes expresaron haber recibido información acerca de los métodos de planificación familiar, y de estas 118 (84.3\%) decidieron utilizar métodos de planificación. Los métodos de planificación familiar escogidos por las pacientes fueron dispositivo intrauterino 33 (23.6\%), esterilización quirúrgica 27 (19.3\%), anticonceptivos orales $26(18.6 \%)$, inyección trimestral $26(18.6 \%)$ y condón 5 (3.6\%). Las pacientes calificaron el servicio de ultrasonido como bueno $249(71.1 \%)$, regular 44 (12.6\%), malo $14(4.0 \%) ; 40$ (11.4\%) no contestaron ya que no habían utilizado el servicio.

A la vez, de las 350 pacientes el $92.0 \%$ (322) percibió que la atención brindada por los médicos del servicio era buena,
Cuadro 1. Percepción de los servicios brindados, usuarias externas en la atención en Ginecología y Obstetricia, Instituto Hondureño de Seguridad Social, Tegucigalpa, 2016-2017, n=350.

\begin{tabular}{|c|c|c|c|}
\hline \multirow[b]{2}{*}{ Servicios brindados } & \multicolumn{3}{|c|}{$\begin{array}{l}\text { Percepción de las usuarias externas sobre los } \\
\text { servicios brindados }\end{array}$} \\
\hline & $\begin{array}{l}\text { Bueno } \\
\mathrm{N}(\%)\end{array}$ & $\begin{array}{l}\text { Regular } \\
\mathbf{N}(\%)\end{array}$ & $\begin{array}{l}\text { Malo } \\
\mathrm{N}(\%) \\
\end{array}$ \\
\hline $\begin{array}{l}\text { Información sobre cuidados después de } \\
\text { la consulta }\end{array}$ & $290(82.9)$ & $43(12.3)$ & $17(4.9)$ \\
\hline Privacidad en la consulta médica & $246(70.3)$ & $95(21.1)$ & $9(2.6)$ \\
\hline Comodidad ofrecida en la consulta & $290(82.9)$ & $48(13.7)$ & $12(3.4)$ \\
\hline Limpieza en el consultorio & $285(81.4)$ & $51(14.6)$ & $14(4.0)$ \\
\hline Aseo de los sanitarios & $201(57.4)$ & $130(37.1)$ & $16(4.6)$ \\
\hline Atención en la farmacia & $65(18.6)$ & $125(35.7)$ & $159(45.4)^{\star}$ \\
\hline Orientación por parte de enfermería & $244(69.7)$ & $80(22.9)$ & $20(5.7)$ \\
\hline $\begin{array}{l}\text { Explicación del estado de salud por } \\
\text { parte del médico }\end{array}$ & $280(80.0)$ & $48(13.7)$ & $17(4.9)^{\star *}$ \\
\hline
\end{tabular}

*Una paciente no contestó $(0.3 \%) ;{ }^{*}$ Cinco pacientes no contestaron (1.4\%). 
$6.6 \%(23.1)$ que era regular y $1.4 \%(4.9)$ que era mala. El $84.3 \%$ (295) opinó que el personal de enfermería brindó buena atención, el $12.8 \%$ (45) regular y $2.8 \%$ (10) atención mala. Dentro del otro personal que brindó algún servicio (farmacia, laboratorio, aseo, vigilancia), el $74.6 \%$ (261) expresó que la atención fue buena, $19.7 \%$ (69) regular y $5.7 \%$ (20) mala (ver Cuadro 2).

La percepción de las pacientes acerca de los servicios que se deben incrementar en atención, fue contar con más especialidades $7.4 \%$ (26), servicio de radiología 3.1\% (11), mamografía $2.3 \%(8)$, más personal para realizar ultrasonidos $1.7 \%(6)$, Nutrición 1.1\% (4), contar con maternidad 1.1\% (4), más cirugías $0.8 \%$ (3). La percepción de las pacientes acerca de los aspectos que se deben mejorar en el servicio fue el contar con más médicos $73.0 \%$ (255), más medicamentos $73.0 \%$ (255), disminuir el tiempo de espera $26.0 \%$ (91), mejorar la atención de farmacia $21.0 \%$ (73), mejorar el tiempo entre citas $21.0 \%$ (73) y crear ventanillas para embarazadas $7.0 \%$ (24) (ver Cuadro 3 ).

Cuadro 2. Percepción del trato recibido de acuerdo al personal que brindó la atención, usuarias externas en la atención en Ginecología y Obstetricia, Instituto Hondureño de Seguridad Social, Tegucigalpa, 2016-2017, n=350.

\begin{tabular}{lccc}
\hline Personal que brindó la atención & \multicolumn{3}{c}{$\begin{array}{c}\text { Percepción de las usuarias externas } \\
\text { sobre el trato recibido }\end{array}$} \\
& $\begin{array}{c}\text { Buena } \\
\text { N (\%) }\end{array}$ & $\begin{array}{c}\text { Regular } \\
\text { N (\%) }\end{array}$ & Mala \\
N (\%) \\
\hline Médico & $322(92.0)$ & $23(6.6)$ & $5(1.4)$ \\
Enfermera & $295(84.3)$ & $45(12.8)$ & $10(2.8)$ \\
Otro personal $^{*}$ & $261(74.6)$ & $69(19.7)$ & $20(5.7)$ \\
\hline
\end{tabular}

*Otro personal: Farmacia, Laboratorio, vigilancia, aseo.

Cuadro 3. Percepción sobre el tipo de servicio que se debe incrementar y los aspectos que se deben mejorar, usuarias externas en la atención en Ginecología y Obstetricia, Instituto Hondureño de Seguridad Social, Tegucigalpa, 2016$2017, \mathrm{n}=350$.

\begin{tabular}{lc}
\hline Tipo de Servicio & $\begin{array}{c}\text { Usuarias externas } \\
\mathbf{N}(\%)\end{array}$ \\
\hline Servicios a incrementar & $26(7.4)$ \\
Especialidades & $11(3.1)$ \\
Rayos X & $8(2.3)$ \\
Mamografía & $6(1.7)$ \\
Más personal que realice ultrasonidos & $4(1.1)$ \\
Nutrición & $4(1.1)$ \\
Maternidad & $3(0.8)$ \\
Cirugías & $288(82.5)$ \\
Ninguno & \\
Aspectos a mejorar & \\
Contratar más médicos & $255(73.0)$ \\
Disponibilidad de medicamentos & $255(73.0)$ \\
Disminuir los tiempos de espera & $91(26.0)$ \\
Mejorar la atención en la farmacia & $73(21.0)$ \\
Disminuir el tiempo entre citas & $73(21.0)$ \\
Ventanilla exclusiva para embarazadas & $24(7.0)$ \\
& \\
\hline
\end{tabular}

\section{DISCUSIÓN}

La encuesta de satisfacción aplicada en la Clínica Periférica No. 1 del IHSS, Tegucigalpa, se basó en parámetros como la conformidad del usuario con los servicios prestados y la percepción sobre las características de la atención recibida como si fue rápido y suficiente. Utilizando como medida una gradación de bueno a malo, se mostró en los resultados que la mayoría de las puntuaciones brindadas consideraron el grado de "bueno". En el caso de las respuestas en relación al tratamiento brindado en el consultorio médico $(80.0 \%)$, orientación por parte del servicio de enfermería (69.7\%), atención en el servicio de ultrasonido ginecológico/obstétrico $(71.1 \%)$, parámetros como la privacidad en la consulta $(70.3 \%)$ y el ambiente físico (81.4\% ambiente "limpio"). El servicio que no recibió calificación de "bueno" fue el servicio de farmacia, contando con porcentajes bajos (18.6\%). Varios reportes internacionales de diferentes organizaciones enfatizan que mejorar la calidad de los cuidados de salud es necesario para alcanzar las coberturas efectivas de salud universal, definiendo calidad del cuidado como aquel efectivo en mantener o mejorar la salud y centrado en la persona, respetuoso y responsable con las preferencias individuales, necesidades y valores. ${ }^{6}$ La experiencia del paciente es un indicador dentro del proceso y refleja los aspectos interpersonales de la calidad del cuidado que recibe. En contraste, satisfacción del paciente es una medida de resultado de las experiencias del cuidado junto con los resultados y confianza en el sistema de salud..$^{1,8}$

Instituciones como la OMS, el Instituto de Medicina de Estados Unidos y la Organización para la Cooperación y el Desarrollo Económico (OCDE) sugieren que un sistema de salud debe considerar mejoras en seis áreas o dimensiones de la calidad de la atención en salud para obtener buenos resultados en los cuidados que se otorgan a los usuarios del sistema sanitario. Estas dimensiones son efectividad, eficiencia, oportunidad, atención centrada en el paciente, equidad y seguridad..$^{9}$ En el momento actual cobra gran importancia el tema de la calidad en los servicios de salud, proceso que de una u otra forma se ha venido desarrollando en el Sistema Nacional de Salud y ahora con mayor vigencia pues en varias unidades se está aplicando este modelo. .0,11 $^{1}$

La necesidad de mejorar las interacciones de las mujeres con los proveedores de asistencia sanitaria sigue siendo fundamental para garantizar la calidad del servicio. Parte integral de este proceso es la adopción de las directrices clínicas y normas profesionales, que formen una visión para un sistema de salud que está centrado en las personas, sensible y eficaz para ambos. ${ }^{12}$ La satisfacción de los usuarios externos que expresa un juicio de valor individual y subjetivo es el aspecto clave de la definición y valoración de la calidad y es el indicador más usado en los servicios de salud. ${ }^{13}$ Entre las encuestas validadas, unas de las más utilizadas son SERVQUAL y SERVPERF, que evalúan aspectos como expectativas y percepciones de los usuarios en el primer caso y calidad tomando en cuenta las percepciones que los usuarios se forman cuando hacen uso de un servicio en 
particular en la segunda. ${ }^{14}$ La encuesta utilizada en el presente estudio es similar a SERVQUAL, tomando en cuenta aspectos como orientación brindada por el personal, atención con rapidez, trato amable, entre otros. La calidad es una secuencia de actividades que relacionan al prestador de los servicios con el usuario, identificando tres aspectos interrelacionados entre sí: calidad, calidez y oportunidad de la atención. La calidad de la atención incluye elementos tales como la secuencia de actividades que relacionan al prestador de los servicios con el usuario en cuanto a oportunidad de la atención, accesibilidad a la unidad, tiempo de espera y resultados de la intervención clínica. La calidez en la atención se refiere al trato cordial, atento y con información que se proporciona al usuario del servicio. ${ }^{15} \mathrm{La}$ oportunidad en la atención se refiere a la ocurrencia de la atención médica en el momento que se requiera y la realización de lo que se debe hacer con la secuencia adecuada., ${ }^{5,13}$

Desde hace más de dos décadas conocer la calidad con que se otorgan los servicios médicos es mencionado como el primer paso para mejorar la prestación de los mismos. ${ }^{10}$ Se han descrito cinco elementos fundamentales: excelencia profesional, uso eficiente de los recursos, mínimo riesgo para el paciente, alto grado de satisfacción e impacto final en la salud. ${ }^{16} \mathrm{De}$ igual forma, la relación paciente proveedor se ha identificado como factor que influencia el éxito de los servicios externos de consulta y programas especializados como es el caso del cuidado de pacientes VIH positivos y la adherencia al tratamiento. ${ }^{17,18}$ Otro aspecto que los sistemas de salud deben considerar en la búsqueda de la satisfacción del usuario en cuanto a los servicios brindados como otro ingrediente en la fórmula de éxito, es el relacionado a la mejora en la vida laboral de los cuidadores y el ambiente que promueva el disfrute de la vida laboral lo cual ha demostrado ser esencial para crear sistemas de alta calidad en los servicios. ${ }^{19,20}$

Este estudio pudo haber reflejado mejor la percepción de las pacientes usuarias si la selección de las participantes hubiese sido aleatoria y durante un mayor tiempo para incrementar el tamaño muestral. En conclusión, las pacientes usuarias del Servicio de Ginecología y Obstetricia de la Clínica Periférica No. 1 se encuentran mayormente satisfechas de la atención recibida, esperan que se incrementen los servicios como radiología, número de especialidades y la contratación de personal con el fin de disminuir tiempos de espera para estudios y consulta. Se recomienda poder profundizar en las razones por las cuales los servicios son percibidos como regulares y malos, lo cual requiere otro tipo de instrumentos. Estos tipos de estudios son importantes ya que contribuyen a la mejora continua de la atención en salud.

\section{CONTRIBUCIONES}

ALC concibió la idea y diseñó el estudio. Ambas autoras coordinaron la recolección de la información y su análisis. Ambas autoras redactaron el artículo, incorporaron las ediciones editoriales y aprobaron la versión final del mismo.

\section{AGRADECIMIENTOS}

Al Instituto Hondureño de Seguridad Social, Clínica Periférica No. 1 y su personal, por toda la atención brindada para la realización de este estudio. A los médicos residentes del Postgrado de Ginecología y Obstetricia, Facultad de Ciencias Médicas UNAH, rotando por la Clínica Periférica No. 1 en el período diciembre 2016 a enero 2017, por la aplicación de las encuestas a las pacientes participantes en el estudio.

\section{REFERENCIAS}

1. Reyes-Morales H, Flores-Hernández $\mathrm{S}$, Sauceda-Valenzuela AL, VértizRamírez JJ, Juárez-Ramírez C, Wirtz Veronika J, et al. Percepción de los usuarios sobre la calidad de la atención ambulatoria en servicios de salud en México. Salud Pública Méx Méx [Internet]. 2013 [acceso junio 2019]; 55 (Supl 2): S100-S105. Disponible en: http://www.scielo.org.mx/scielo. php?script=sci_arttext\&pid=S0036-36342013000800005\&lng=es _

2. Pérez Pulido MO, Orlandoni Merli G, Ramoni Perazzi J, Valbuena Vence M. Percepción de la calidad en la prestación de servicios de salud con un enfoque seis sigma. Rev Cubana Salud Pública [Internet]. 2018 [acceso junio 2019];44(2):325-343. Disponible en: http://scielo.sld.cu/scielo. php?script=sci_arttext\&pid=S0864-34662018000200325\&lng=es

3. Robledo Galván H, Meljem Moctezuma J, Fajardo Dolci G, Olvera López D. De la idea al concepto en la calidad en los servicios de salud. CONAMED [Internet]. 2012 [acceso junio 2019];17(4):172-175. Disponible en: https:// www.medigraphic.com/cgi-bin/new/resumen.cgi?IDARTICULO=57702

4. Giovanella L, Almeida PF de, Vega Romero R, Oliveira S, Tejerina Silva H. Panorama de la Atención Primaria de Salud en Suramérica: concepciones, componentes y desafíos. Saúde debate[Internet]. 2015[acceso junio 2019]; 39(105): 300-322. Disponible en: http://www.scielo.br/scielo. php?script=sci_arttext\&pid=S0103-11042015000200300\&lng=en

5. Sasche M, Sesia P, Pintado A, Lastra Z. Calidad de la atención obstetrica desde la perspectiva de derechos, equidad e interculturalidad en centros de salud de Oaxaca. CONAMED [Internet] 2012 [ acceso junio 2019]; 17(Supl. 1): S4-S15. Disponible en: https:/www.medigraphic.com/cgi-bin/ new/resumen.cgi?IDARTICULO=57682

6. Dois A, Contreras A, Bravo P, Mora I, Soto G, Solís C. Principios orientado- res del modelo integral de salud familiar y comunitario desde la perspectiva de los usuarios. Rev Med Chile [Internet]. 2016 [acceso junio 2019];144 (5):585-592. Disponible en: https://scielo.conicyt.cl/scielo.php?script=sci_ arttext\&pid=S0034-98872016000500005\&lng=es

7. Bermúdez Madriz JL, Sáenz MR, Muiser J, Acosta M. Sistema de salud de Honduras. Salud Publ Mex [Internet]. 2011 [acceso junio 2019]; 53(Supl 2): S209-S219. Disponible en: https://www.scielosp.org/article/spm/2011. v53supp12/s209-s219/es/

8. Aguirre Gas H. Evaluación de la calidad de Atención. Expectativas de los pacientes y de los trabajadores de las unidades médicas. Salud Publ Mex [Internet]. 1990 [acceso junio 2019];32(2):170-180. Disponible en: http:// saludpublica.mx/index.php/spm/article/view/5285/5435

9. Puchi-Gómez C, Paravic-Klijn T, Salazar A. Indicadores de calidad de la atención en salud en hospitalización domiciliaria: revisión integradora. Aquichan [Internet]. 2018 [acceso junio 2019]; 18(2): 186-197. Disponible en: http://www.scielo.org.co/pdf/aqui/v18n2/1657-5997-aqui-18-02-00186. pdf

10. Ramos Domínguez BN. Control de calidad de la atención de salud [Internet]. 2a ed. La Habana: Editorial Ciencias Médicas; 2011. [acceso junio 2019]. Disponible en: http://files.sld.cu/scap/files/2012/01/control_calidad. pdf

11. Saturno Hernández PJ, Hernández Avila M, Magaña Valladares L, Garcia Saisó S, Vertiz Ramírez JJ. Estrategia integral de formación para la mejora continua de la calidad de los servicios de salud. Salud Públ Méx [Internet]. 2015 [acceso junio 2019]; 57 (3):275-283. Disponible en: http://www.scielo.org.mx/scielo.php?script=sci_arttext\&pid=S0036- 
$36342015000300017 \&$ Ing=es

12. Organización Panamericana de la Salud. Normas mundiales para mejorar la calidad de los servicios de atención de salud de los adolescentes. Guía de aplicación de un enfoque fundamentado en las normas para mejorar la calidad de los servicios de salud prestados a los adolescentes. Vol. 1, Normas y criterios. [Internet]. Washington DC: OPS, 2016. [acceso junio 2019]. Disponible en: http://iris.paho.org/ xmlui/bitstream/handle/123456789/28569/9789275319048_v1-spa. pdf?sequence=1\&isAllowed=y

13. Salas Perea RS. Propuesta de estrategia para la evaluación del desempeño laboral de los médicos en Cuba. Educ Med Super [Internet]. 2010 [acceso junio 2019];24(3):387-417. Disponible en: http://scielo.sld.cu/scielo. php?script=sci_arttext\&pid=S0864-21412010000300011\&Ing=es

14. Cabello E, Chirinos JL. Validación y aplicabilidad de encuestas SERVQUAL modificadas para medir la satisfacción de usuarios externos en servicios de salud. Rev Med Hered [Internet]. 2012 [acceso junio 2019];23(2):88-95. Disponible en: http://www.scielo.org.pe/scielo. php?script=sci_arttext\&pid=S1018-130X2012000200003\&lng=es

15. Schwartzmann L. Calidad de vida relacionada con la salud: aspectos conceptuales. Cienc Enferm [Internet]. 2003 [acceso junio 2019];9(2):921. Disponible en: https://scielo.conicyt.cl/scielo.php?script=sci_
arttext\&pid=S0717-95532003000200002\&Ing=es

16. Massip Pérez C, Ortiz Reyes RM, Llantá Abreu MC, Peña Fortes M, Infante Ochoa I. La evaluación de la satisfacción en salud: un reto a la calidad. Rev Cubana Salud Pública [Internet]. 2008 [acceso junio 2019];34(4). Disponible en: http://scielo.sld.cu/scielo.php?script=sci_arttext\&pid=S086434662008000400013\&lng=es

17. Herrera C, Campero L, Caballero M, Kendall T. Relación entre médicos y pacientes con VIH: influencia en apego terapéutico y calidad de vida. Rev. Saúde Pública [Internet] 2008 [acceso junio 2019];42(2):249-255. Disponible en: http://www.scielo.br/scielo.php?script=sci_arttext\&pid=S003489102008000200009\&Ing=en

18. Odallo $B$, Opondo $E$, Onyango M. Litigating to ensure access to quality maternal health care for women and girls in Kenya. Reproduct Health Matters. 2018; 26 (53): 123-129.

19. Jacobi J. Farmacéuticos clínicos: profesionales esenciales del equipo de atención clínica. Rev Med Clin Las Condes. 2016; 27(5): 578-584.

20. Rodríguez-Villamizar LA, Vera-Cala LM, Rivera R, Rojas OA, Uribe LM. Evaluación de percepción de competencias de trabajadores respecto del Modelo Integral de Atención en Salud. Rev Univ Ind Santander Salud [Internat]. 2017 [acceso junio 2019]; 49 (2): 302-311. Disponible en: http://www.scielo.org.co/scielo.php?script=sci_arttext\&pid =S0121-08072017000200302\&

ABSTRACT. Background: The concept of quality of care in health services implies that patients are informed and share decisionmaking for their care. Objective: To establish the level of user satisfaction in the Obstetrics and Gynecology Service, Peripheral Clinic No. 1, Honduran Institute of Social Security (IHSS), Tegucigalpa, 2016-2017. Methods: We use the User Satisfaction Survey of the Modified Health Care Insurance Scheme, created by the IHSS (Year 2014). The survey was carried out by Postgraduate Gynecology and Obstetrics Residency Program, after obtaining written informed consent. Results: A total of 350 patients were interviewed. Age of patients interviewed were $18-35$ years in $61.0 \%$ (215). The admission procedures were perceived as rapid by $46.0 \%(163)$. The $92.0 \%$ (322) and $84.0 \%$ (295) were satisfied with the care provided by medical and nursing staff, respectively. The information about care after the medical appointment was perceived as good $82.9 \%$ (290), regular $12.3 \%$ (43) and bad $4.9 \%$ (17). The privacy in the clinic was perceived as good $70.3 \%$ (246), regular $21.1 \%(95)$ and bad $2.6 \%(9)$. In regard to the comfort offered during the appointment, it was perceived as good $82.9 \%$ (290), regular $13.7 \%$ (48) and bad $3.4 \%$ (12). Discussion: A substantial proportion of the external users interviewed considered the care provided to be good and noted that they would like to expand the service offering. Continuous improvement of health care is important.

Keywords: Ambulatory care, Delivery of health care, Evaluation studies, Health services accessibility, Quality of health care. 